Pacific

Journal of

Mathematics

MONOTONICITY AND SYMMETRY RESULTS FOR DEGENERATE ELLIPTIC EQUATIONS ON NILPOTENT LIE GROUPS

I. Birindelli And J. Prajapat 


\title{
MONOTONICITY AND SYMMETRY RESULTS FOR DEGENERATE ELLIPTIC EQUATIONS ON NILPOTENT LIE GROUPS
}

\author{
I. Birindelli AND J. Prajapat
}

In this paper we prove some monotonicity results for solutions of semilinear equations in nilpotent, stratified groups. We also prove a partial symmetry result for solutions of nonlinear equations on the Heisenberg group.

\section{Introduction.}

Berestycki and Nirenberg (see e.g., [2]) introduced the so called "sliding method" to prove monotonicity results for semilinear elliptic equations in convex domains of $\mathbf{R}^{n}$. The idea here is to implement the method in the general setting of nilpotent stratified groups. Let us mention that examples of such groups include the Heisenberg group and, of course, the Euclidean space. Hence, in particular, we obtain monotonicity results for a large class of degenerate elliptic semilinear equations.

More precisely, let $(G, \circ)$ be a nilpotent, stratified Lie group, see Section 2 for definitions and properties. Clearly the notion of "convexity" has to be related to the group action:

Definition 1.1. Fix $\eta \in G$. A domain $\Omega \subset G$ is said to be $\eta$-convex (or convex in the direction $\eta$ ) if for any $\xi_{1} \in \Omega$ and any $\xi_{2} \in \Omega$ such that $\xi_{2}=\alpha \eta \circ \xi_{1}$ for some $\alpha>0$, we have $s \eta \circ \xi_{1} \in \Omega$ for every $s \in(0, \alpha)$.

Observe that this coincides with the notion of convexity in a given direction for domains in the Euclidean space. Any Koranyi ball in the Heisenberg group $H^{n}=\left(\mathbf{R}^{2 n+1}, \circ\right)$ is an example of a domain which is $\eta$-convex for any $\eta \in H^{n}$.

At the end of the paper we show a "cube" in the Heisenberg group $H^{1}$, which is obtained by sliding a square of the plane $x_{1}=0$ through the group action in the direction of $(0,1,0)$. In the figure, we have shaded the top and bottom surfaces in order to make the cube more visible. Observe that this set is convex in both the directions $e_{1}=(1,0,0)$ and $e_{2}=(0,1,0)$.

Let

$$
\Delta_{G}=\sum_{i=1}^{m} X_{i}^{2}
$$


denote the sub-Laplacian operator defined on $G$ and let $S_{2}^{Q}$ denote the Sobolev space for the group $G$ where $Q$ is the homogeneous dimension of $G$; see details in Section 2. For $\eta \in G$ and $u \in S_{2}^{Q}$, let $\mathcal{T}_{\eta} u(\xi):=u(\eta \circ \xi)$.

Our main result is the following:

Theorem 1.1. Let $(G, \circ)$ be a stratified, nilpotent Lie group and $\Omega$ be an arbitrary bounded domain of $G$ which is $\eta$-convex for some $\eta \in G$. Let $u \in S_{2}^{Q}(\Omega) \cap C(\bar{\Omega})$ be a solution of

$$
\begin{cases}\Delta_{G} u+f(u)=0 & \text { in } \Omega \\ u=\phi & \text { on } \partial \Omega\end{cases}
$$

where $f$ is a Lipschitz continuous function. Assume that for any $\xi_{1}, \xi_{2} \in \partial \Omega$, such that $\xi_{2}=\alpha \eta \circ \xi_{1}$ for some $\alpha>0$, we have for each $s \in(0, \alpha)$

$$
\phi\left(\xi_{1}\right)<\mathcal{T}_{s \eta} u\left(\xi_{1}\right)<\phi\left(\xi_{2}\right) \text { if } s \eta \circ \xi_{1} \in \Omega
$$

and

$$
\phi\left(\xi_{1}\right)<\mathcal{T}_{s \eta} \phi\left(\xi_{1}\right)<\phi\left(\xi_{2}\right) \text { if } s \eta \circ \xi_{1} \in \partial \Omega .
$$

Then $u$ satisfies

$$
\mathcal{T}_{s_{1} \eta} u(\xi)<\mathcal{T}_{s \eta} u(\xi)
$$

for any $0<s_{1}<s<\alpha$ and for every $\xi \in \Omega$.

Moreover, $u$ is the unique solution of $(1.1)$ in $S_{2}^{Q}(\Omega) \cap C(\bar{\Omega})$ satisfying (1.2).

Remark. Clearly, (1.4) implies that $u$ is monotone along $\gamma(s)=s \eta \circ \xi$. Observe that the curve $\gamma$ is the integral curve of a right invariant vector field $R_{\eta}$, even though the operator $\Delta_{G}$ is left invariant.

An immediate consequence of Theorem 1.1 is:

Corollary 1.2. Under the assumptions of Theorem 1.1, if $f$ is $C^{1}$ and $R_{\eta}$ commutes with $\Delta_{G}$ then

$$
R_{\eta} u>0 \text { in } \Omega \text {. }
$$

In [1], L. Almeida and Y. Ge have proved monotonicity results in the general setting of manifolds. However, they consider solutions of uniformly elliptic semilinear equations.

An important tool in the proof of Theorem 1.1 is the "Maximum principle in domains with small measure" which is new in the setting of degenerate elliptic equations. On the other hand, it is known for uniformly elliptic and parabolic operators (see [1] , [2], [6]) and it has found extensive applications, see for e.g., [3] and [15]. 
Using the notations of [14], on a bounded domain $\Omega \subset \mathbf{R}^{N}$, consider the operator

$$
L u(x)=\frac{1}{2} \sigma^{i k}(x)\left(\sigma^{j k}(x) u_{x^{j}}(x)\right)_{x^{i}}+b^{i}(x) u_{x^{i}}(x)
$$

where $\sigma^{k}=\left(\sigma^{i k}\right), k=1, \ldots, n_{1}$, and $b=\left(b^{i}\right)$ are smooth vector fields given on $\mathbf{R}^{N}$ and $n_{1}$ is an integer. We assume that the Lie algebra generated by the family of vector fields $\left\{b, \sigma^{k}, k=1, \ldots, n_{1}\right\}$ has dimension $N$ at all points in the closure $\bar{D}$ of a neighborhood $D$ of $\bar{\Omega}$. Equivalently, $L$ is an operator satisfying Hörmander condition.

Similarly to [2], we say that the maximum principle holds for the operator $L+c$ where $c$ is an $L^{\infty}$ function in $\Omega$ if for $u \in S_{2}^{Q}(\Omega)$

$$
L u+c(\xi) u \geq 0 \text { in } \Omega
$$

and

$$
\varlimsup_{\xi \rightarrow \partial \Omega} u(\xi) \leq 0
$$

implies that $u \leq 0$ in $\Omega$. Note that by embedding theorems (see e.g., [17]), $u \in S_{2}^{Q}(\Omega)$ implies that $u$ is continuous in $\Omega$.

The following proposition is the maximum principle for "domains with small measure" of $\mathbf{R}^{N}$ for the operators $L$ :

Proposition 1.3 (Maximum Principle). Let $\Omega$ be a bounded domain in $\mathbf{R}^{N}$ and $L$ be an operator as defined above and $c$ such that $c(\xi) \leq b$ in $\Omega$ for some $b \in \mathbf{R}^{+}$. There exists $\delta>0$, depending only on $N$ and $b$, such that the maximum principle holds for $L+c$ in $\Omega$ provided

$$
\text { meas }(\Omega)<\delta \text {. }
$$

A weak comparison principle was derived in [1] using a Poincaré type inequality. An anonymous referee raised the question of whether we could similarly use a Poincaré type inequality to give an alternative proof of Proposition 1.3. In the last section, we derive a Poincaré type inequality for subelliptic operators and as a consequence of this inequality, we give an alternative proof of Proposition 1.3. We thank the referee for pointing this out.

Note that the class of operators $L$ defined in (1.6) and the sub-Laplacian $\Delta_{G}$ associated with a nilpotent Lie group $G$ are examples of subelliptic operators (see (6.1) for the definition of a subelliptic operator). Since it is possible to associate a group structure with the operator $L$ in (1.6) (see [19]), the monotonicity result Theorem 1.1 is infact true for a more general nilpotent Lie group. We have given the result here for nilpotent, stratified Lie group to avoid technical details. However, it may not be possible to associate a general subelliptic operator with a group structure. 
In Section 2 we state the basic definitions concerning nilpotent stratified Lie groups in general and the Heisenberg group in particular, in Section 3 we prove Proposition 1.3 and in Section 4 we prove Theorem 1.1.

Section 5 is a different application of the maximum principle in domains with small measure i.e., Proposition 1.3. We prove a symmetry result for positive "cylindrical" solutions of semilinear equations in a class of bounded symmetric domains in the Heisenberg group under some conditions. The generalization of Gidas, Ni, Nirenberg result (see [10]), to the Heisenberg Laplacian is a difficult open problem. Theorem 5.1 is a step towards the solution of this problem.

Finally in Section 6 we prove a Poincaré type inequality as mentioned above.

\section{Preliminaries.}

In this section we recall the basic notions of nilpotent, stratified Lie groups from $[\mathbf{1 9}]$. Let $(\mathcal{G},[]$,$) be a real finite dimensional Lie algebra, \mathcal{G}_{1}=\mathcal{G}$ and $\mathcal{G}_{i}=\left[\mathcal{G}, \mathcal{G}_{i-1}\right]$ for $i \geq 2$. Then $\left\{\mathcal{G}_{i}\right\}_{i \geq 2}$ is a decreasing sequence of Lie sub-algebras of $\mathcal{G}$. The Lie algebra $\mathcal{G}$ is said to be nilpotent of rank $r$ if $\mathcal{G}_{r+1}=0$. A Lie group $G$ is said to be nilpotent of rank $r$ if its Lie algebra is nilpotent of rank $r$.

A stratified group $G$ is a simply connected nilpotent group whose Lie algebra $\mathcal{G}$ admits a direct sum decomposition (as vector space)

$$
\mathcal{G}=V_{1} \oplus \ldots \oplus V_{m}
$$

with $\operatorname{dim} V_{j}=n_{j},\left[V_{1}, V_{j}\right]=V_{j+1}$ for $1 \leq j<m$ and $\left[V_{1}, V_{m}\right]=0$. Thus $V_{1}$ generates $\mathcal{G}$ as a Lie algebra.

More precisely, given a Lie algebra $(\mathcal{G},[\cdot])$ satisfying the above conditions, consider $\mathbf{R}^{N}$ where $N=\sum_{j=1}^{l} n_{j}$ with the group operation $\circ$ given by the Campbell-Hausdorff formula

$$
\eta \circ \xi=\eta+\xi+\frac{1}{2}[\eta, \xi]+\frac{1}{12}[\eta,[\eta, \xi]]+\frac{1}{12}[\xi,[\xi, \eta]]+\ldots
$$

Note that since $\mathcal{G}$ is nilpotent there are only a finite number of nonzero terms in the above sum; precisely those involving commutators of $\xi$ and $\eta$ of length less than $m$. Then $(G, \circ)=\left(\mathbf{R}^{N}, \circ\right)$ is the nilpotent, stratified group whose Lie algebra of left-invariant vector fields coincides with the Lie $\operatorname{algebra}(\mathcal{G},[]$,$) .$

Consider the standard basis $e_{1}, \ldots, e_{n_{1}}$ of the subspace $\mathbf{R}^{n_{1}}$ of $G$. Let $X_{1}, \ldots, X_{n_{1}}$ denote the corresponding "coordinate vector fields", i.e.,

$$
X_{i}(f)(\xi)=\lim _{t \rightarrow 0} \frac{f\left(\xi \circ t e_{i}\right)-f(\xi)}{t},
$$

for any smooth function $f$ defined on $G$ and for $i=1, \ldots, n_{1}$. The family $\left\{X_{1}, \ldots, X_{n_{1}}\right\}$ forms a basis for $V_{1}$. We define the sub-Laplacian operator 
on $G$ as

$$
\Delta_{G}=\sum_{i=1}^{n_{1}} X_{i}^{2} .
$$

We observe that this operator is subelliptic and satisfies Hörmander's condition. Hence the Bony's maximum principle holds (see [5]). Furthermore, the vector fields are invariant with respect to the group action, viz,

$$
X_{i} \circ \mathcal{T}_{\eta}=\mathcal{T}_{\eta} \circ X_{i}
$$

and clearly so is the operator $\Delta_{G}$. In fact, this is a fundamental property of the operator which we shall use to prove Theorem 1.1.

Since the vector fields $\left\{X_{1}, \ldots, X_{n_{1}}\right\}$ generate $\mathcal{G}$ as Lie algebra, we can define recursively for $j=1, \ldots, m$, and $i=1, \ldots, n_{j}$, a basis $\left\{X_{i, j}\right\}$ of $V_{j}$ as

$$
\begin{aligned}
X_{i, 1} & =X_{i}\left(i=1, \ldots, n_{1}\right) \\
X_{\alpha} & =\left[X_{i_{1}},\left[X_{i_{2}}, \ldots,\left[X_{i_{j-1}}, X_{i_{j}}\right]\right] \ldots\right]
\end{aligned}
$$

with $\alpha=\left(i_{1}, \ldots, i_{j}\right)$ multi-index of length $j$ and $X_{i_{k}} \in\left\{X_{1}, \ldots, X_{n_{1}}\right\}$. With the decomposition $\mathcal{G}=\mathbf{R}^{n_{1}} \oplus \ldots \oplus \mathbf{R}^{n_{m}}$, we define a parameter group of dilations $\delta_{\lambda}$ by setting for

$$
\begin{gathered}
\xi=\xi_{1}+\ldots+\xi_{m}, \quad\left(\xi_{i} \in \mathbf{R}^{n_{i}}\right) \\
\delta_{\lambda}(\xi)=\sum_{i=1}^{m} \lambda^{i} \xi_{i} .
\end{gathered}
$$

For any $\xi \in G$, the Jacobian of the map $\xi \mapsto \delta_{\lambda}(\xi)$ is $\lambda^{Q}$ where

$$
Q=\sum_{i=1}^{m} i n_{i}
$$

The integer $Q$ is called the homogeneous dimension of $G$. Note that the euclidean dimension of $G$ is $N=\sum_{i=1}^{m} n_{i}$. We have $Q \geq N$ with equality in the trivial case $m=1$ and $G=\mathbf{R}^{n_{1}}$.

Observe that since $G$ is simply connected, the exponential map exp : $\mathcal{G} \rightarrow$ $G$ is a diffeomorphism and the Lebesgue measure on $\mathcal{G}, d x=d x_{1} \ldots d x_{N}$, pulled back to $G$ by the map $\exp ^{-1}$, is left and right invariant with respect to the group action.

We recall that the equivalent of the Sobolev spaces, as introduced by Folland and Stein $[\mathbf{8}, \mathbf{9}]$, are

$$
S_{2}^{q}(\Omega)=\left\{f \in L^{q}(\Omega) \text { such that } X^{I} f \in L^{q}(\Omega) \text { for }|I| \leq 2\right\},
$$


where $I:=\left(\alpha_{1}, \ldots, \alpha_{h}\right)\left(\alpha_{i} \leq n_{1}\right)$ denotes a multi-index of length $|I|=h$ and $X^{I}=X_{\alpha_{1}} \ldots X_{\alpha_{h}}$. The norm in $S_{2}^{q}$ is given by:

$$
\|u\|_{S_{2}^{q}}^{q}=\int_{\Omega}\left(\sum_{|I|=1}^{2}\left|X^{I} u\right|^{q}+|u|^{q}\right) d \xi
$$

A typical example of a nilpotent, stratified Lie group is the Heisenberg group $H^{n}=\left(\mathbf{R}^{2 n+1}, \circ\right)$ endowed with the group action $\circ$ defined by

$$
\xi_{0} \circ \xi=\left(x+x_{0}, y+y_{0}, t+t_{0}+2 \sum_{i=1}^{n}\left(x_{i} y_{0_{i}}-y_{i} x_{0_{i}}\right)\right) \text {. }
$$

Here we denote the elements of $H^{n}$ either by $(z, t) \in \mathbb{C}^{n} \times \mathbf{R}$ or $(x, y, t) \in$ $\mathbf{R}^{n} \times \mathbf{R}^{n} \times \mathbf{R}$ where $z=x+i y, x=\left(x_{1}, \ldots, x_{n}\right), y=\left(y_{1}, \ldots, y_{n}\right)$.

The Lie algebra of $H^{n}$ decomposes as $\mathbf{R}^{2 n} \oplus \mathbf{R}$. Hence $n_{1}=2 n, n_{2}=1$ and the anisotropic norm which is homogeneous with respect to the dilation given in (2.3) is defined by

$$
|\xi|_{H}=\left(\left(x^{2}+y^{2}\right)^{2}+t^{2}\right)^{\frac{1}{4}} .
$$

The so called Koranyi ball is the set: $\left\{\xi \in H^{n}\right.$ such that $|\xi|_{H} \leq$ const $\}$.

The generating vector fields are defined by

$$
\begin{array}{ll}
X_{i}=\frac{\partial}{\partial x_{i}}+2 y_{i} \frac{\partial}{\partial t}, & \text { for } i=1, \ldots, n, \\
X_{n+i}:=Y_{i}=\frac{\partial}{\partial y_{i}}-2 x_{i} \frac{\partial}{\partial t}, & \text { for } i=1, \ldots, n .
\end{array}
$$

Furthermore, we have

$$
X_{(i, j+n)}:=\left[X_{i}, Y_{j}\right]=-4 \delta_{i, j} T
$$

for $1 \leq i, j \leq n$ and $T:=\frac{\partial}{\partial t}$. Also, observe that the homogeneous dimension of $H^{n}$ is $2 n+2$, which is strictly greater than its linear dimension.

\section{Maximum principle.}

The Proposition 1.3 is a consequence of the following theorem by Krylov:

Theorem 3.1. Let $L$ be an operator defined as in (1.6), on a smooth bounded domain $D \subset \mathbf{R}^{N}$. For a fixed $\epsilon \in(0,1)$ and $f \in L_{p}(D)$ with any $p \in(1, \infty)$, let $u=: R f \in W_{p}^{2}(D)$ be the unique solution of the equation

$$
(L+\epsilon \Delta) u-u=f
$$

with zero boundary condition. Here $\Delta$ is the Laplace operator on $\mathbf{R}^{N}$. Then, there exists a (large) $p_{0} \in(1, \infty)$ and a (small) $\alpha \in(0,1)$ both independent 
of $\epsilon$ and such that for any $p \geq p_{0}$, subdomain $D_{1} \subset \overline{D_{1}} \subset D$, and $f \in L_{p}(D)$ we have

$$
\begin{gathered}
\sup _{D}|R f| \leq C\|f\|_{L_{p}(D)}, \\
|R f(x)-R f(y)| \leq C|x-y|^{\alpha}\|f\|_{L_{p}(D)},
\end{gathered}
$$

where the constants $C$ are independent of $x, y, f$, and $\epsilon$.

We refer to [14] for a beautiful proof of this result. Also, it follows from [13] that one does not need the condition that the domain $D$ is smooth in the above theorem.

Proof of Proposition 1.3. Define $u^{+}(x)=\max \{u(x), 0\}$. To prove the proposition, we need to show that $u^{+} \equiv 0$. Let $\Omega^{+}=\{x \in \Omega: u(x)>0\}$. Then $u^{+}$satisfies the equation

$$
L u^{+}(x)+c(x) u^{+}(x) \geq 0
$$

for $x \in \Omega^{+}$and

$$
u^{+}=0
$$

on the boundary $\partial \Omega^{+}$.

Now let $v$ be the solution of the equation

$$
L v-v=-u^{+}-b u^{+}
$$

on $\Omega^{+}$with zero boundary condition. From Theorem 3.1 we have

$$
\sup _{\Omega^{+}} v \leq C(b+1)\left\|u^{+}\right\|_{L_{p}\left(\Omega^{+}\right)} .
$$

But from (3.3), (3.4) and $u^{+}-v=0$ on $\partial \Omega$, the maximum principle implies that $u^{+} \leq v$ in $\Omega^{+}$. Hence it follows that

$$
\sup _{\Omega} u^{+} \leq C(b+1)\left\|u^{+}\right\|_{L_{p}\left(\Omega^{+}\right)} .
$$

Estimating the r.h.s. we have

$$
\sup _{\Omega} u^{+} \leq C(b+1) \text { meas }(\Omega)^{1 / p} \sup _{\Omega} u^{+} .
$$

Hence, if we choose $\delta$ such that $C(b+1) \delta<1$, then meas $(\Omega)<\delta$ and (3.5) implies that $u^{+} \equiv 0$ i.e., $u \leq 0$ in $\Omega$.

\section{Proof of Theorem 1.1.}

As in previous sections $(G, \circ)=\left(\mathbf{R}^{N}, \circ\right)$ is a nilpotent, stratified Lie group and $\Delta_{G}$ is the corresponding sub-Laplacian operator. Using the notations of Theorem 1.1, define $u_{s}(\xi)=\mathcal{T}_{s \eta} u(\xi)$ for $s>0$. The function $u_{s}$ is defined on the domain $\Omega_{s}=\{\xi \in G: s \eta \circ \xi \in \Omega\}$, obtained by "translation" of $\Omega$. 
Furthermore, since the sub-Laplacian is invariant under the group action it follows that $u_{s}$ satisfies the equation

$$
\Delta_{G} u_{s}+f\left(u_{s}\right)=0 \text { in } \Omega_{s} .
$$

Since the domain $\Omega$ is bounded, there exists $s_{0}>0$ such that $\Omega_{s_{0}} \cap \Omega=\emptyset$ and for $s<s_{0}$ near $s_{0}, \Omega_{s} \cap \Omega \neq \emptyset$. And as we slide the domain $\Omega_{s}$, i.e., we decrease $s$ to zero, we get $\Omega_{0}=\Omega$. Now for $s<s_{0}$ consider the function $w_{s}=u_{s}-u$ in $D_{s}=\Omega_{s} \cap \Omega$. Clearly, to prove (1.4), we need to show that

$$
w_{s}>0 \text { for every } 0<s<s_{0} \text {. }
$$

Observe that $w_{s}$ satisfies the equation

$$
\Delta_{G} w_{s}+c_{s}(\xi) w_{s}=0 \text { in } D_{s},
$$

where $c_{s}$ is a $L^{\infty}$ function satisfying $\left|c_{s}(\xi)\right| \leq C$ for $\xi \in D_{s}$ for all $s$. Furthermore, due to the assumptions (1.2) and (1.3) we have

$$
w_{s} \geq 0
$$

on the boundary of $D_{s}$.

Let $\delta$ denote the constant appearing in the Proposition 1.3 corresponding to the operator $\Delta_{G}$ defined on $\Omega$. For $s>s_{1}$ and sufficiently near $s_{1}$, meas $\left(D_{s}\right)<\delta$. Therefore, by Proposition 1.3 it follows that

$$
w_{s} \geq 0
$$

in $D_{s}$ for $s$ near $s_{1}$. Moreover, (1.2), (1.3) and the strong maximum principle implies that

$$
w_{s}>0 \text { in } D_{s} \text {. }
$$

Let $\mu_{1}=\min \left\{\mu: w_{s}>0\right.$ for every $\left.s>\mu\right\}$. We claim that $\mu_{1}=0$. Suppose, by contradiction that $\mu_{1}>0$. Then $w_{\mu_{1}} \geq 0$. Since $\mu_{1}>0$, again the strong maximum principle implies that $w_{\mu_{1}}>0$ in $D_{\mu_{1}}$.

Choose a compact set $\Sigma \subset D_{\mu_{1}}$ such that meas $\left(D_{\mu_{1}} \backslash \Sigma\right)<\delta / 3$, where $\delta$ is as fixed above. Since $\Sigma$ is compact, for $s<\mu_{1}$ with $\mu_{1}-s$ small, we have

$$
w_{s} \geq 0 \text { in } \Sigma \text {. }
$$

Further, for $0<\mu<\mu_{1}$ and sufficiently close to $\mu_{1}$, we have

$$
\Sigma \subset D_{\mu} \text { and meas }\left(D_{\mu_{1}} \backslash \Sigma\right)<\delta \text {. }
$$

Fix a $\mu<\mu_{1}$ such that (4.1) and (4.2) hold for all $s, \mu<s<\mu_{1}$. Proposition 1.3 implies $w_{s} \geq 0$ in $D_{s} \backslash \Sigma$ for $\mu<s<\mu_{1}$. This, together with (4.1) implies that $w_{s} \geq 0$ on $D_{s}$ for all $s, \mu<s<\mu_{1}$. Since $s>\mu>0$ and $w_{s} \not \equiv 0$, we further conclude from the strong maximum principle that $w_{s}>0$ in $D_{s}$ for all $s, \mu<s<\mu_{1}$; which contradicts the definition of $\mu_{1}$. Hence $\mu_{1}=0$ and therefore (1.4) holds true.

Uniqueness. To prove the uniqueness, suppose $u, v \in S_{2}^{Q}(\Omega) \cap C(\bar{\Omega})$ are two solutions of (1.1). Consider the function $w_{s}(\xi):=v_{s}(\xi)-u(\xi)$ in $\Omega_{s} \cap \Omega$ 
where $v_{s}(\xi)=v(s \eta \circ \xi)$. We can go through the above proof with this function to conclude that for every $\xi \in \Omega$,

$$
u(\xi)<v(s \eta \circ \xi) \text { for } s>0 .
$$

Similarly, considering the function $\widetilde{w}_{s}(\xi):=u_{s}(\xi)-v(\xi)$ in $\Omega_{s} \cap \Omega$ we have for every $\xi \in \Omega$ that

$$
v(\xi)<u(s \eta \circ \xi) \text { for } s>0 .
$$

Letting $s \rightarrow 0$ in (4.3) and (4.4), it follows

$$
u \equiv v \text { in } \Omega .
$$

The proof of the Corollary 1.2 is immediate. Observe that since $f$ is $C^{1}$ and $R_{\eta}$ commutes with $\Delta_{G}$, then $R_{\eta} u$ satisfies the equation

$$
\Delta_{G} R_{\eta} u+f^{\prime}(u) R_{\eta}(u)=0
$$

in $\Omega$. Furthermore, Theorem 1.1 implies that $R_{\eta} u \geq 0$ in $\Omega$. But $R_{\eta} u \not \equiv 0$ in $\Omega$. Hence the maximum principle implies that $R_{\eta} u>0$ in $\Omega$.

\section{A symmetry result.}

We begin by defining a special class of functions and domains in the Heisenberg group:

Definition 5.1. We say that a function $u$ defined on $H^{n}$ is cylindrical if there exists $\xi_{o} \in H^{n}$ such that $v(\xi):=u\left(\xi_{o} \circ \xi\right)$ is a function depending only on $(r, t)$, where $r=\left(x^{2}+y^{2}\right)^{\frac{1}{2}}$. We say that a domain $\mathcal{C} \subset H^{n}$ is a cylinder if there exists a cylindrical function $\Phi$ such that $\xi \in \mathcal{C} \Leftrightarrow \Phi(\xi)<0$.

Observe that a Koranyi ball is a cylinder. Also, the Euclidean ball $\left\{(z, t) \in H^{n}:|z|^{2}+t^{2} \leq\right.$ constant $\}$ with center at the origin belongs to this class. However, a Euclidean ball centered at a point other than the origin need not be a cylinder in $H^{n}$.

In this section we prove a symmetry result for positive, cylindrical solutions of semilinear equations defined on a "cylinder" (as defined above) in the Heisenberg group $H^{n}$. The proof relies on the maximum principle in domains with small measure and the adaptation of the moving plane method to $H^{n}$. This method was used for the first time in the setting of the Heisenberg group in [4].

In the rest of the section, without loss of generality we will assume that $\xi_{o}$ occurring in the definition (5.1) is 0 . 
Theorem 5.1. Let $\mathcal{C}$ be a bounded cylinder in $H^{n}$ defined by a function $\Phi$. Let $u \in C^{2}(\mathcal{C}) \cap C(\overline{\mathcal{C}})$ be a positive, cylindrical solution of the equation

$$
\begin{aligned}
\Delta_{H} u+f(u) & =0 \text { in } \mathcal{C} \\
u & =0 \text { on } \partial \mathcal{C}
\end{aligned}
$$

where $f$ is a Lipschitz function. If $\Phi(r, t)=\Phi(r,-t)$ then $u(r, t)=u(r,-t)$ on $\mathcal{C}$.

Proof. The proof relies on the adaptation of the moving plane method to $H^{n}$. Let $T_{\lambda}=\left\{\xi \in H^{n}: t=\lambda\right\}$ denote the hyperplane orthogonal to the $t$ direction and let $R_{\lambda}(x, y, t)=(y, x, 2 \lambda-t)$ denote the $H$-reflection (see [4]). We shift the plane from infinity towards the domain, i.e., we decrease $\lambda$ until it reaches the value $\lambda_{0}$ such that the plane $T_{\lambda_{0}}$ "touches" the boundary $\partial \mathcal{C}$.

For $\lambda<\lambda_{0}$, let $D_{\lambda}=\{(x, y, t) \in \mathcal{C}: t>\lambda\}$ be the subset of $\mathcal{C}$ cut off by the plane $T_{\lambda}$. Define $u_{\lambda}=u \circ R_{\lambda}$ on $D_{\lambda}$. Since $u$ is cylindrical, so is $u_{\lambda}$ and further $u_{\lambda}(r, t)=u(r, 2 \lambda-t)$. Moreover, since $\Delta_{H}$ is invariant with respect to the $H$-reflection (see [4]), it follows that $u_{\lambda}$ satisfies Equation (5.1) in $D_{\lambda}$.

Now consider the function $w_{\lambda}=u_{\lambda}-u$ in $D_{\lambda}$. It satisfies the equation

$$
\Delta_{H} w_{\lambda}+c(\xi) w_{\lambda} \leq 0 \text { in } D_{\lambda}
$$

with the boundary conditions

$$
w_{\lambda} \geq 0 \text { on } \partial D_{\lambda} .
$$

Let $\delta$ be the constant appearing in Proposition 1.3 corresponding to the operator $L=\Delta_{H}+c$ of Equation (5.3) on $\mathcal{C}$. Observe that here $c(\xi)$ is bounded since $f$ is Lipschitz. For $\lambda<\lambda_{0}$ and sufficiently close to $\lambda_{0}$, we have meas $\left(D_{\lambda}\right)<\delta$. Hence by the maximum principle 1.3 , it follows that $w_{\lambda} \geq 0$ in $D_{\lambda}$.

We claim that $w_{\lambda} \geq 0$ in $D_{\lambda}$ for every $\lambda>0$. For otherwise, let $\mu=$ $\inf \left\{\lambda: w_{s} \geq 0\right.$ for $\left.\lambda<s<\lambda_{0}\right\}$ and suppose $\mu>0$. By continuity, $w_{\mu} \geq 0$. Further since $u$ is positive inside $\Omega$, the maximum principle implies that $w_{\mu}>0$ in $D_{\mu}$.

Let $K \subset D_{\mu}$ be a compact set such that

$$
\text { meas }\left(D_{\mu} \backslash K\right)<\frac{\delta}{2}
$$

where $\delta$ is the constant chosen above. Since $K$ is compact and $w_{\mu}>0$ on $K$, there exists $\bar{\lambda}$ near $\mu$ and $0<\bar{\lambda}<\mu$ such that

$$
w_{\bar{\lambda}}>0 \quad \text { in } K \text {. }
$$

Further we may choose $\bar{\lambda}$ such that

$$
\text { meas }\left(D_{\bar{\lambda}} \backslash K\right)<\delta \text {. }
$$

On $D_{\bar{\lambda}} \backslash K, w_{\bar{\lambda}}$ satisfies the differential equation (5.3) with boundary condition $w_{\bar{\lambda}} \geq 0$ on $\partial\left(D_{\bar{\lambda}} \backslash K\right)$. Since meas $\left(D_{\bar{\lambda}} \backslash K\right)<\delta$, by Proposition 1.3 it 
follows that $w_{\bar{\lambda}} \geq 0$ on $D_{\bar{\lambda}} \backslash K$. Therefore, $w_{\bar{\lambda}} \geq 0$ on $D_{\bar{\lambda}}$. From (5.5), the strong maximum principle implies that $w_{\bar{\lambda}}>0$ in $D_{\bar{\lambda}}$. This contradicts the definition of $\mu$. Hence $\mu=0$; which completes the proof.

Remark. It is clear from the proof that we don't use the fact that the solutions $u$ are cylindrical, we only use that $u(x, y, t)=u(y, x, t)$. Hence the theorem holds true under this weaker condition on the solution. Almeida and $\mathrm{Ge}([\mathbf{1}])$ use a similar condition, precisely if $x=\left(x_{1}, \ldots, x_{n}\right)$ and $y=$ $\left(y_{1}, \ldots, y_{n}\right)$ they suppose that $u\left(x_{1}, x_{2}, \ldots, y_{1}, y_{2}, \ldots, t\right)=u\left(y_{1}, x_{2}, \ldots, x_{1}\right.$, $\left.y_{2}, \ldots, t\right)$.

\section{A Maximum principle for locally subelliptic operators.}

Here we prove a maximum principle for locally subelliptic operators, using the idea suggested by T. Coulhon. We first recall the definition of subelliptic operator from [18] and [12].

An operator $L$ is said locally subelliptic in $\mathbf{R}^{n}$ if for an open subset $\Omega$ of $\mathbf{R}^{n}$, we can write

$$
L=\sum_{i, j=1}^{n} \frac{1}{h(x)} \frac{\partial}{\partial x_{i}}\left(h(x) a_{i j}(x) \frac{\partial}{\partial x_{j}}\right)
$$

where the coefficients $a_{i j}$ and $h$ are $C^{\infty}$ real valued functions on $\bar{\Omega}, h$ is positive and the matrix $A(x)=\left(a_{i j}(x)\right)$ is symmetric positive semidefinite for every $x \in \bar{\Omega}$.

Further $L$ satisfies a subelliptic estimate: There exists a constant $C$ and a number $\varepsilon>0$ such that all $u \in C_{0}^{\infty}(\Omega)$ satisfy

$$
\|u\|_{\varepsilon}^{2} \leq C\left(\int \sum_{i, j=1}^{n} a_{i j}(x) \frac{\partial u}{\partial x_{i}} \frac{\partial u}{\partial x_{j}} h(x) d x+\int|u(x)|^{2} d x\right)
$$

where

$$
\|u\|_{s}=\left(\int|\hat{u}(\xi)|^{2}\left(1+|\xi|^{2}\right)^{s} d \xi\right)^{1 / 2}
$$

denotes the standard Sobolev norm of order $s$.

Clearly, if $A$ is a positive definite matrix, then $L$ is an elliptic operator which satisfies (6.2) with $\varepsilon=1$. Examples of $L$ include the operators which can be written as sum of vector fields satisfying Hörmander's condition. In this case, $\varepsilon=1 / 2$. See $[\mathbf{1 2}]$ for other examples.

Let $\rho$ denote the distance function canonically associated with $L$ which is continuous and defines a topology on $\mathbf{R}^{n}$ (see [12] and references therein). We denote this space $\mathcal{M}=\left(\mathbf{R}^{n}, \rho\right)$. 
The gradient associated to operator $L$ is defined as

$$
\nabla_{L}(u, v)=\frac{1}{2} L(u v)+u L v+v L u
$$

see $[\mathbf{1 8}]$. We denote

$$
\left|\nabla_{L} u\right|=\left(\nabla_{L}(u, u)\right)^{1 / 2}
$$

Almeida and Ge proved a weak comparison principle (Theorem 2.1) in [1] for $n$-dimensional manifolds $(M, g)$ for the elliptic operator defined locally as

$$
\mathcal{L} u=-\sum_{i, j=1}^{\infty} \frac{1}{(\operatorname{det} g)^{1 / 2}} \frac{\partial}{\partial x^{i}}\left(a^{\prime}\left(|\nabla u|^{2}\right)(\operatorname{det} g)^{1 / 2} g^{i j} \frac{\partial u}{\partial x^{j}}\right)
$$

where $a \in W^{2, \infty}((0, \infty)) \cap C^{0}([0, \infty))$ is such that $a^{\prime}(t)-2\left(a^{\prime}(t)\right)^{-} t \geq \alpha>0$ for some $\alpha>0$.

Their proof relied on the following Poincaré type inequality: For an open subset $M^{\prime}$ of $M$, there exists two constants $\gamma, C>0$ such that if $\operatorname{vol}\left(M^{\prime}\right) \leq$ $\gamma$, then

$$
\int_{M^{\prime}}|\psi|^{2} d \operatorname{vol} \leq C \operatorname{vol}\left(M^{\prime}\right)^{2 / n} \int_{M^{\prime}}|\nabla \psi|^{2} d \operatorname{vol}, \text { for all } \psi \in H_{0}^{1}\left(M^{\prime}\right) .
$$

We will essentially show that an inequality similar to (6.4) holds for the operator $L$ on $\mathcal{M}$.

Proposition 6.1. Let $B_{L}(\xi, R) \subset \mathcal{M}$ denote a ball with center $\xi$ and radius $R$ (with respect to the distance $\rho$ ). Then for every nonempty compact subset $\Omega$ of $B(\xi, R)$, there exists $\nu>0$ and a constant $C_{0}$ depending only on $B(\xi, R)$ such that

$$
\|f\|_{2}^{2} \leq C_{0} \text { meas }(\Omega)^{\nu}\left\|\nabla_{L} f\right\|_{2}^{2} \quad \text { for every } f \in C_{0}^{\infty}(\Omega) .
$$

Here, $\nabla_{L}$ is the gradient associated to $L$.

Observe that when $L$ is an elliptic operator, then (6.5) reduces to (6.4) with $\nu=2 / n$.

Proof. Observe that, the distance function $\rho$ satisfies the doubling property (see [12]): There exists a constant $d$ such that

$$
\left|B_{L}(x, 2 R)\right| \leq d\left|B_{L}(x, R)\right| \text { for all } x \in \mathcal{M}, R>0
$$

where $\left|B_{L}(x, R)\right|=\mu\left(B_{L}(x, R)\right)$ is the volume or Lebesgue measure of the ball $B_{L}(x, R)$.

We also recall the Poincaré inequality proved in [18] (Lemma 2.4): There exists constant $C$ such that for every $f \in C_{0}^{\infty}(M)$

$$
\left\|f-f_{R}\right\| \leq C R\|\nabla f\|_{2} \text { for all } R>0,
$$

where $f_{R}$ is the mean of $f$ over the ball $B_{L}(x, R)$. 
Now as in [11], [18], [7] (see references therein) it can be proved that (6.7) and (6.6) implies the Faber-Krahn type of inequality for $\mathcal{M}$ : i.e., there exists constants $a>0, \nu>0$ such that, for every $x \in \mathcal{M}, R>0$ and for every nonempty compact subset $\Omega$ contained in $B_{L}(x, R)$,

$$
\lambda_{1}(\Omega) \geq \frac{a}{R^{2}}\left(\frac{\left|B_{L}(x, R)\right|}{|\Omega|}\right)^{\nu}
$$

where

$$
\lambda_{1}(\Omega)=\inf \left\{\frac{\left\|\nabla_{G} f\right\|_{2}^{2}}{\|f\|_{2}^{2}}: f \in C_{0}^{\infty}(\Omega)\right\} .
$$

In particular, we can conclude from Faber-Krahn inequality (6.8) that for a fixed ball $B_{L}(x, R), R \geq 1 / 2$, for every nonempty subset $\Omega \subset B_{L}(x, R)$, we have

$$
\begin{aligned}
\|f\|_{2}^{2} & \leq \frac{R^{2}}{a}\left(\frac{|\Omega|}{\left|B_{L}(x, R)\right|}\right)^{\nu}\left\|\nabla_{G} f\right\|_{2}^{2} \text { for every } f \in C_{0}^{\infty}(\Omega) \\
& =C_{0}|\Omega|^{\nu}\left\|\nabla_{G} f\right\|_{2}^{2} \text { for every } f \in C_{0}^{\infty}(\Omega)
\end{aligned}
$$

where $C_{0}=\frac{R^{2}}{a\left|B_{L}(x, R)\right|^{\nu}}$ is a fixed constant for $B_{L}(x, R)$.

Using the inequality (6.5) we have:

Proposition 6.2. Let $\Omega$ be a bounded domain in $\mathcal{M}$ and $L$ be a subelliptic operator as defined in (6.1). Assume that $\|c\|_{L^{\infty}(\Omega)} \leq b$. For a subset $\Sigma \subset \Omega$, there exists $\delta>0$ depending only on $b, \Omega$ and $C_{0}$ (the constant appearing in (6.5)) such that the maximum principle holds for $L+c$ in $\Sigma$ provided

$$
\text { meas }(\Sigma)<\delta \text {. }
$$

Proof. First choose a ball $B_{L}\left(x_{0}, R\right)$ such that $\bar{\Omega} \subset B_{L}\left(x_{0}, R\right)$ and fix it for the following discussion. Note that this $R$ depends on $\Omega$. And let $C_{o}$ be the constant defined in the Proposition 6.1 with respect to this ball.

Let $\Sigma \subset \Omega$ and consider the function $u \in S^{1,2}(\mathcal{M}) \cap L^{\infty}(\mathcal{M})$ satisfying

$$
L u+c(x) u \geq 0 \text { in } \Sigma, \quad \varlimsup_{x \rightarrow \partial \Sigma} u(x) \leq 0 .
$$

Here $S^{1,2}(\mathcal{M})$ is the completion of $C^{1}(\mathcal{M})$ under the seminorm

$$
\|f\|_{1,2}=\left\|\nabla_{L} f\right\|_{2}+\|f\|_{2} .
$$

Define $u^{+}(x)=\max \{u(x), 0\}$ and $\Sigma^{+}=\{x \in \Sigma: u(x)>0\}$. Then $u^{+}$ satisfies the equation

$$
\begin{aligned}
L u^{+}(x)+c(x) u^{+}(x) & \geq 0 \text { in } \Sigma^{+} \\
u^{+} & =0 \text { on } \partial \Sigma^{+} .
\end{aligned}
$$


Multiplying (6.11) by $u^{+}$and integrating by parts, we have

$$
\begin{aligned}
\int_{\Sigma^{+}}\left|\nabla_{L} u^{+}\right|^{2} d x & =-\int_{\Sigma^{+}} c(x)\left|u^{+}\right|^{2} d x \\
& \leq\|c\|_{L^{\infty}} \int_{\Sigma^{+}}\left|u^{+}\right|^{2} d x \leq b \int_{\Sigma^{+}}\left|u^{+}\right|^{2} d x .
\end{aligned}
$$

Now from (6.13) and (6.5) we obtain

$$
\int_{\Sigma^{+}}\left|\nabla_{L} u^{+}\right|^{2} d x \leq b \int_{\Sigma^{+}}\left|u^{+}\right|^{2} d x \leq b C_{0}|\Sigma|^{\nu} \int_{\Sigma^{+}}\left|\nabla_{L} u^{+}\right|^{2} d x .
$$

Choose $\delta<\left(b C_{0}\right)^{-1 / \nu}$. If meas $(\Sigma)<\delta$ then (6.14) implies that

$$
\int_{\Sigma^{+}}\left|\nabla_{L} u^{+}\right|^{2} d x=0
$$

It follows that the inequalities in (6.14) are in fact equalities with each term equal to 0 . In particular,

$$
\int_{\Sigma^{+}}\left|u^{+}\right|^{2} d x=0
$$

and hence $u^{+} \equiv 0$.

Acknowledgements. We would like to thank Xavier Cabré and Arvind Nair for useful conversations.

We also thank Thierry Coulhon for fruitful discussion and suggesting the references related to the inequalities in Section 6 .

This work was completed while the second author was visiting the Mathematics Department of Università degli studi di Roma "La Sapienza" with a grant from G.N.A.F.A.-CNR. She thanks the Department of Mathematics for the hospitality. 


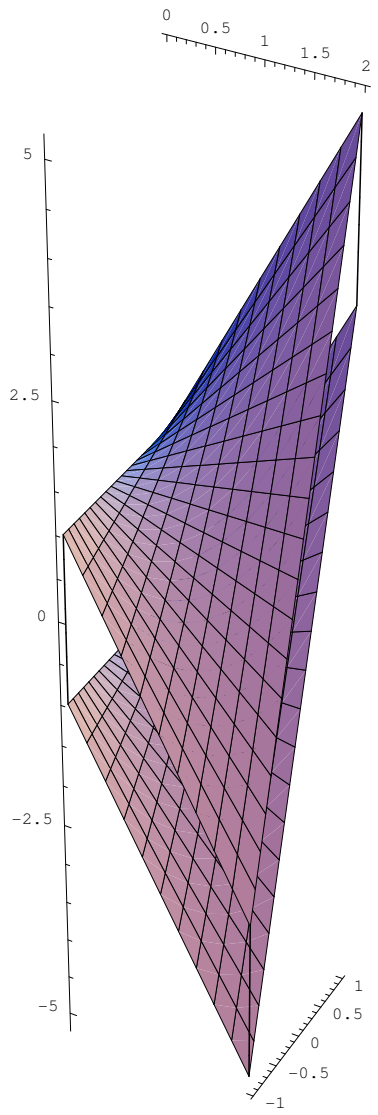

A "cube" in the Heisenberg group $H^{1}$.

\section{References}

[1] L. Almeida and Y. Ge, Symmetry results for positive solutions of some elliptic equations on manifolds, Ann. Global Anal. Geom., 18(2) (2000), 153-170, MR 2001b:58059, Zbl 0968.58015.

[2] H. Berestycki and L. Nirenberg, On the method of moving planes and the sliding method, Bol. Soc. Bras. Mat., 22 (1991), 1-37, MR 93a:35048, Zbl 0784.35025.

[3] H. Berestycki, L. Nirenberg and S.R.S. Varadhan, The principal eigenvalue and maximum principle for second order elliptic operator in general domains, Comm. Pure Appl. Math., 47 (1994), 47-92, MR 95h:35053, Zbl 0806.35129.

[4] I. Birindelli and J. Prajapat, Nonlinear Liouville theorems in the Heisenberg group via the moving plane method, Comm. Partial Differ. Equat., 24(9-10) (1999), 1875-1890, MR 2000k:35055, Zbl 0944.35023. 
[5] J.M. Bony, Principe du Maximum, Inégalité de Harnack et unicité du problème de Cauchy pour les operateurs elliptiques dégénérés, Ann. Inst. Fourier Grenobles, 19(1) (1969), 277-304, MR 41 \#7486, Zbl 0176.09703.

[6] Xavier Cabré, Inequalities for solutions of Elliptic and Parabolic equations, Comm. Pure Appl. Math., XLVIII (1995), 539-570, MR 96c:35023, Zbl 0828.35017.

[7] T. Coulhon and A. Grigoryan, Random walks on graphs with regular volume growth, GAFA, 8 (1998), 656-701, MR 99e:60153, Zbl 0918.60053.

[8] G.B. Folland, Fundamental solution for subelliptic operators, Bull. Amer. Math. Soc., 79 (1979), 373-376, MR 47 \#3816, Zbl 0256.35020.

[9] G.B. Folland and E.M. Stein, Estimates for the $\partial_{b}$ complex and anlysis on the Heisenberg group, Indiana Univ. Math. J., 41 (1992), 71-97.

[10] B. Gidas, W.M. Ni and L. Nirenberg, Symmetry and related properties via the maximum principle, Comm. Math. Phys., 68 (1979), 209-243, MR 80h:35043, Zbl 0425.35020.

[11] A. Grigoryan, The heat kernel upper bounds on a complete noncompact manifold, Revista Math. Iber., 10(2) (1994), 395-452, MR 96b:58107, Zbl 0810.58040.

[12] D. Jerison and A. Sanchez-Calle, Subelliptic second order differential operators, Proceedings, Univ. of Md., Lecture notes in Math., 1277, Springer, Berlin (1986), 47-77, MR 89b:35021, Zbl 0634.35017.

[13] R. Khasminskii, N. Krylov and N. Moshchuk, On parameters estimation for linear stochastic differential equations, Probab. Theory Relat. Fields, 113(3) (1999), 443472, MR 2000f:62192.

[14] N.V. Krylov, Holder continuity and $L_{p}$ estimates for elliptic equations under general Hörmander's condition, Topological methods on Nonlinear Analysis, 9(2) (1997), 249-258, MR 99b:35077, Zbl 0892.35066.

[15] S. Kumaresan and J. Prajapat, Analogue of Gidas-Ni-Nirenberg result for domains in hyperbolic space and sphere, Rend. Ist. Mat. Univ. Trieste, 30(1-2) (1998), 107-112, MR 2000d:35074, Zbl 0930.35072.

[16] Guozhen Lu, Weighted Poincaré and Sobolev inequalities for vector fields satisfying Hörmander's condition and applications, Revista Math. Iber., 8(3) (1992), 367-439, MR 94c:35061, Zbl 0804.35015.

[17] L. Saloff-Coste, Théorèmes de Sobolev et inégalités de Trudinger sur certains groupes de Lie, C. R. Acad. Sci. Paris, Série I, 306 (1988), 305-308, MR 89h:22013, Zbl 0661.43005 .

[18] _ A note on Poincaré, Sobolev and Harnack inequalities, IMRN, 2 (1992), 27-38, MR 93d:58158, Zbl 0769.58054.

[19] N. Varopoulos, L. Saloff-Coste and T. Coulhon, Analysis and geometry on groups, Cambridge tracts in Mathematics, 100, Cambridge University Press, 1992, MR 95f:43008.

Received January 31, 2000 and revised July 6, 2001. 
Università DI Roma "LA SAPIENZA"

P.LE Aldo Moro 5

00185 Roma

ITALIA

Indian Statistical Institute

8Th Mile, Mysore Road

BANGALORE 560059

INDIA

E-mail address: jyotsna@isibang.ac.in 